\title{
A novel cucumber albino mutant caused by chloroplast development deficiency
}

\section{Jinqiang Yan}

Guangdong Academy of Agricultural Sciences

\section{Bin Liu}

Shanghai Jiao Tong University

\section{Zhenqiang Cao}

Guangdong Academy of Agricultural Sciences

\section{Piaoyun Sun}

Guangdong Academy of Agricultural Sciences

\section{Wenrui Liu}

Guangdong Academy of Agricultural Sciences

\section{Zhaojun Liang}

Guangdong Academy of Agricultural Sciences

\section{Min Wang}

Guangdong Academy of Agricultural Sciences

\section{Qingwu Peng}

Guangdong Academy of Agricultural Sciences

\section{Lin Chen}

Guangdong Academy of Agricultural Sciences

Yu'e Lin

Guangdong Academy of Agricultural Sciences

Biao Jiang ( $\sim$ jiangbiao198354@163.com )

Guangdong Academy of Agricultural Sciences

\section{Xiaoming He}

Guangdong Academy of Agricultural Sciences

\section{Research article}

Keywords: Albino, mutant, chloroplast, deficiency, transcriptome

Posted Date: April 6th, 2020

DOI: https://doi.org/10.21203/rs.3.rs-20991/v1 
License: (c) (i) This work is licensed under a Creative Commons Attribution 4.0 International License. Read Full License 


\section{Abstract}

Background Photosynthesis is a fundamental process for plant growth and development dependent on a precise network, including formation of chloroplast and chlorophyll synthesis. Chloroplast development deficiency could lead to albinism in higher plant.

Results Here, we report a cucumber albino recessive mutant that processed white cotyledons under light condition and is unable to produce first true leaf. Meanwhile, albino mutant could grow out creamy green cotyledons under dark condition but died after exposing to light. Using fluorescence microscopy and transmission electron microscope (TEM), impaired chloroplasts were observed. We identified 7 and 3 differentially expressed genes (DEG) involved in Chlorophyll metabolism and Methylerythritol 4phosphate (MEP) pathway through transcriptome analysis, respectively. We also examined the reported homologous genes for albino mutants from other plants. Two of 12 genes, TOC159 and DXS1, were upregulated in cucumber albino mutants as well. The reliability of RNA sequencing results were further confirmed by real-time quantitative PCR (qPCR).

Conclusions Taken together, we elaborate the differences between albino mutant and normal seedlings from a single cucumber progeny. This mutant is a new material to study protoplast development.

\section{Background}

As a DNA containing organelle, Chloroplast plays crucial roles in attuning plant development and plant interaction with environmental cues. Chloroplast is originated from proplastids in cotyledons at the time of illumination via the process of photomorphogenesis $(1,2)$. Chloroplast, consisted with thylakoids stacked into defined grana, is the site of photosynthesis, production of hormones (i.e., abscisic acid, jasmonic acid and salicylic acid), and major metabolism (3). Its abnormal development or accumulation of pigment content could affect photosynthesis thus disrupt plant growth and biomass yield (4-7).

Leaf color mutation has been widely reported in many plant species, among which natural or induced albino mutants were frequently identified and characterized, especially in Arabidopsis and rice. Some mutants were environmental dependent albino but conditionally green-revertible. A mutation in OsABCl8 caused plants showing albino leaves under continuous rainy weather with defective chloroplast, but the leaves could turn green gradually after rainy season (8). Mutation in gene OsTCD 5 that encodes a monooxygenase, or OsTCD11 that encodes the ribosomal small subunit protein S6 in chloroplasts (RPS6) were temperature sensitive albino mutant, presenting albino leaves at low temperature and normal green leaves at high temperature (9). Gene FLN2 encoding fructokinase-like protein2 showing opposite performance, with mutation in this gene were with albino leaves at high temperature (10). Many mutations were found only showing albino at certain development stage. Disruption of a pentatricopeptide repeat protein caused albino during seedling stage but the plants were able to turn green accompanying plant development (11). A mutation in SEEDLING PLASTID DEVELOPMENT1 resulted in albino cotyledon but the plants had similar appearance once initial green true leaves developed and transferred to soil (12). 
Somatic albino mutants were also described with genes expression related to chlorophyll biosynthesis and chloroplast development affected in mutated branch leaf $(13,14)$. Still some mutations were described lethal mainly caused by deficiency in chloroplast development. A single T-DNA insertion causing fragment deletion affecting the function of gene DXS1 led to albino phenotype in tomato with premature lethal performance (15). A single nucleotide mutation in the plastid ribosomal protein produced abnormal chloroplast and caused lethal rice seedlings (16).

Cucumber (Cucumis sativus L.) is a worldwide important vegetable crop of Cucurbitacea family. Cucumber albino mutation was only reported by lida and Amano in 1991 (17), which was induced by irradiation. However, no any further studies were carried out since then. Here, we reported a spontaneous mutation of albino mutant from inbred line "g32", which exhibited white cotyledons and hypocotyl, dying before developing any true leaves. Cytological analyses revealed that chlorophyll signal was absent and chloroplast development was disrupted in the mutant. In the present study, we performed the highthroughput RNA sequencing to examine the differentially expressed genes between albino mutant and wild type.

\section{Results}

\section{Phenotypic characterization of a cucumber albino mutant}

We observed a few natural occurring albino seedlings during the seed increase of cucumber inbred line "g32" which is a south china type cucumber. The cotyledons of these albino seedlings are small and absolute white, and of which hypocotyl is short and white and primary root length is short (Fig.1). The albino mutants dried out and died in a few days after emerging from substrate without growing any true leaves.

\section{Inheritance model of albino mutant}

To investigate the inheritance nature of the albino mutant, we planted a total of 123 seeds from the selfpollinated g23 cucumber, of which 116 were germinated (germination rate 94.3\%). Among the germinated seeds, 32 and 84 seedlings present albino and wild type phenotypes which fit the Mendelian ratio for single recessive gene $\left(\chi^{2}=0.414, p=0.520\right.$, Additional file 2: Table S2).

\section{Short-lived chloroplast recovery in albino mutant under dark condition}

Occasionally, we observed some subtle greenish cotyledons in few albino mutants that were grown under the canopy. It draws our attention that albino mutation might be affected by light. We therefore performed three sets of experiments to validate this hypothesis (Fig. 2a). In the first experiment, all the seeds were grown under dark condition all the time. After emerging from substrate, albino mutant presented creamy green cotyledon and completely white hypocotyl and wild type seedlings show yellowish green cotyledon and hypocotyl (Fig. 2b). As continuedly dark environment, both albino mutant and wild type spindled and died without growing first true leaf eventually. In the second experiment, after the seeds emerged from 
substrate, we first let them grow under light condition until we are able to distinguish albino mutant and wild type. Later on, we transferred them under dark condition and found that albino mutant maintained whitish tissues without turning to cream green (Fig. 2c1-2). In the last experiment, the seeds grew under dark condition until seedlings emerging from substrate, and then they were exposed to light (Fig. 2a3). Upwards 5 hours after exposed to light, the green color in the cotyledon of albino mutant started to degrade (Fig. 2d1). The cotyledon contracted and dry out after exposed to light for 30 hours (Fig. 2d2). Therefore, we may conclude that light is a lethal factor for albino mutant and this damage is irreversible.

Most of the albino phenotypes in other plants are lack of chlorophyll. Thus, we examined the chlorophyll content of cotyledon from wild type, albino mutants that grow under light and dark condition using fluorescence microscopy. Much intensive chlorophyll signals were observed in wild type compared with albino mutant that grown under dark conditions (Fig. 2e; Presented in red). As expect, no chlorophyll signals were caught in albino mutants that grow under light condition.

Since most chlorophyll content in plants are presented in chloroplast, we further investigate the chloroplast ultrastructure in cotyledon of wild type and albino mutant that grew under both light and dark condition using transmission electron microscopy. In wild type cotyledon, we observed numerous welldeveloped, crescent-shaped chloroplast with stroma thylakoids, grana thylakoids, starch granules and plastoglobuli within the membranes (Fig. $2 \mathrm{f} 1,4,7$ ). In contrast, the chloroplast in albino mutant decreased dramatically in number and showed abnormal shape that lack of stroma thylakoids and grana thylakoids structure but contained osmiophilic plastoglobulis in inner membrane system (Fig. 2f2,5,8). The albino mutant that grow under dark condition comprised stroma thylakoid as well as osmiophilic plastoglobulis (Fig. 2f3,6,9).

To summarize, the above results indicated that the chloroplast development is impaired in the albino mutant under light condition during seedling development. Moreover, light probably acts as a lethal factor to the albino mutant by interrupting thylakoid biogenesis, as we could observe the presence of thylakoid in the albino mutant grown in dark but not under light condition.

\section{Transcriptome profiling and identifying differentially expressed genes (DEGs) between albino mutant and wild type}

The transcriptomes of cotyledons from albino mutant and wild type were examined using RNA-seq, each with three biological replicates. Overall, $97,869,948$ to $123,516,412$ clean reads were obtained after filtering low quality reads (Table 1). After mapping to the cucumber reference genome $9930 \mathrm{v} 2(17,18)$, in total, 21,664 transcripts were detected. High coefficients among the replicates demonstrated the consistency of the transcriptional changes within each type (Fig. 3a). In total, 1,256 genes were found upregulated and 1,584 were down-regulated in albino mutant compared with wild type ( $|\log 2 \mathrm{FC}| \geq 2$ ) (Fig. 3b, Additional file 3: Table S3). Based on the annotation, DEGs were categorized to GO and KEGG pathway to deeply understand the significant biological processes and pathways between albino mutant and wild type. 2,175 DEGs were classified into $814 \mathrm{GO}$ processes involved in three categories, biological process, cellular component and molecular function (Additional file 4: Table S4), with biological process being 
dominant category. Cellular carbohydrate biosynthetic process (G0:0034637, $p=0.00010)$ and Cellular carbohydrate metabolic process (G0:0044262, $\mathrm{p}=0.00017)$ are the most significantly enriched processes (Additional file 8: Figure S1). As shown in Fig. 4, transmembrane transport (G0:0055085) and transporter activity (GO:0005215) accounted for the majority among the enriched GO terms, including 171 and 196 DEGs, respectively (Additional file 4: Table S4). Totally, 957 DEGs were assigned to 110 KEGG pathways (Additional file 5: Table S5), among which top 20 enriched KEGG pathways were illustrated in Fig. 4. Carbon metabolism (KEGG: csv01200), phenylpropanoid biosynthesis (KEGG: csv00940) and starch and sucrose metabolism (KEGG: csv00500) pathways occupied large proportion, with 69, 50 and 44 DEGs included, respectively (Additional file 5: Table S5).

\section{Differentially expressed genes involved in chlorophyll metabolism}

Porphyrin and chlorophyll metabolism (KEGG: csv00860) was a significantly enriched pathway in KEGG analysis. Many key enzymes involved in this pathway showed distinct expression profile between albino mutant and wild type. Most of genes, including HEMB (Csa2G401270), HEME (Csa4G082410, Csa5G218840), HEMF (Csa4G056670), HEMG (Csa6G007980), CHLG (Csa4G311220) and CAO (Csa6G385090) were slightly higher expressed in albino mutant than in wild type (Fig. 5a, Additional file 6: Table S6). However, POR (Csa4G638340) was down-regulated in albino mutant, presenting opposite pattern to other genes (Fig. 5a, Additional file 6: Table S6).

\section{Differentially expressed genes involved in Methylerythritol 4-phosphate (MEP) pathway}

The methylerythritol 4-phosphate (MEP) pathway is a source for the production of isoprenoid precursors, isopentenyl diphosphate (IPP) and dimethylallyl diphosphate (DMAPP) in photosynthetic eukaryotes(19). As shown in Fig. 5b, there are at least seven key enzymes involved in the MEP pathway, four candidate genes encoded CMS (Csa3G113320, Csa4G049620), CMK (Csa1G600780) and MCS (Csa4G049620) were found down-regulated in albino mutant (Additional file 6: Table S6).

\section{Thylakoid related genes are affected in albino mutant}

Nineteen DEGs belonging to thylakoid related functional activities, i.e., thylakoid (G0:0009579), thylakoid part (GO:0044436) and thylakoid membrane (G0:0042651) were highlighted (Fig. 6, Additional file 3: Table S3). Three PsbPs (Csa2G030040, Csa1G088470 and Csa1G181310) were found up-regulated in albino mutant while other 16 genes PsbR (Csa4G064020), PsaG/PsaK (Csa3G060980, Csa6G525340), $P s b O$ (Csa6G488340), PsaD (Csa3G147780), PsaH (Csa3G483830), PsbP(Csa4G063440), PsaE (Csa2G079660), PsbY (Csa5G592810), PsbW(Csa7G378440), PsaN(Csa6G483300), PsbQ (Csa1G066480, Csa3G414060), PsbX (Csa1G595840), PsaF (Csa1G714680) and PetM (Csa7G075020) were down-regulated in albino mutant (Additional file 3: Table S3).

\section{Homologous genes from albino mutants in other species}

Mutation of FLN1 (fructokinase-like 1) from barley and rice(20-22), RPL21c (chloroplast 50S ribosome protein L21) from arabidopsis and rice(23, 24), EMB (embryo-specific) from arabidopsis(25-28), PDS3 
(phytoene desaturase) from arabidopsis(29), TOC159 (Translocase of chloroplast 159) from arabidopsis(30, 31), DXS1 (1-deoxy-D-xylulose-5-phosphate synthase 1) from tomato(15), and PurD (phosphoribosylamine-glycine ligase) from rice(32) could cause albinism in higher plant. The CDS of homologous genes of albino mutant and wild type were compared based on our transcriptome results. No variant was found among these genes between mutant and wild type. Nearly no gene, except two, TOC159 (Csa4G001670) and DXS1 (Csa3G114510) were up-regulated in the mutant (Additional file 7: Table S7).

\section{Validation of DEG expression by RT-qPCR}

Eighteen DEGs (gene names were listed in Additional file 1: Table S1) with absolute log2(FoldChange) $>3$ were randomly selected for RT-qPCR verification. Genes encoding peroxidase, isocitrate lyase, Glutathione S-transferase, malate synthase and many others were included. The expression of each gene was presented by relative expression $(-\Delta \Delta \mathrm{Ct})$ using normal green seedling as control. The correlation between relative expression value of albino mutant and RNA-seq result (log2FoldChange) was calculated in EXCEL using CORREL function. The correlation factor between RT-qPCR and RNA-seq data was 0.9899, indicating a strong correlation. Overall, RT-qPCR validation indicates the reliability of RNA-seq result.

\section{Discussion}

Albinism happens among different living things varying from human beings, animals as well as higher plants (33-35). In this study, some albino seedlings were observed in the progenies from a self-pollinated cucumber fruit during the breeding selection period of an inbred line named g32. This cucumber albino mutant presented white hypocotyl finally presented lethal performance without developing first true leaf under light condition. In most studies, happening of albino phenotype in plants were caused by absence of chlorophyll and abnormal chloroplast development $(27,36,37)$. Absent chlorophyll signal and defective chloroplast development in albino mutant were in consistent with most albino mutant in this respect. In normal seedlings, ultrastructure of chloroplast is well presented with compactly arranged chloroplasts while few or even no chloroplast structure was seen in albino seedlings. Also, an abnormal chloroplast ultrastructure in albino mutant were observed lacking starch granules and thylakoids, but with osmiophilic plastoglobuli (Fig. 4). Osmiophilic plastoglobuli generally appears as a result of the degradation of thylakoid membranes under stresses (38), therefore we propose that light might act as an abiotic stress cue for albino mutant, leading to the degradation of thylakoid membranes.

Interestingly, we found that this cucumber albino mutant is different to fln1, rpl21c, emb, pds3, toc159, dxs 1 and purd albino mutants ${ }^{15,20-32}$ since it could recover white cotyledon to green under dark condition. Similar phenotype was reported in Arabidopsis albino pap7-1 mutant $(39,40)$. Pap7-1 mutant was with albino cotyledon and finally died grown under light condition but undistinguishable with wild type grown in darkness (40). From the TEM analysis, chloroplasts with thylakoids were observed. Since pap7-1 mutant could be arrested under very dim light (40), we tried to culture the mutant to dark to let it 
develop but finally failed. Still it needs to be proved whether our mutant could produce plant architecture or even flower as pap7-1 grown under sucrose supplemented medium and dim light.

Differences in transcriptional level between albino mutant and wild type were also determined. Key genes involved in chlorophyll metabolism and MEP pathway and thylakoid related genes were differentially accumulated. Genes in chlorophyll metabolism were mainly up-regulated in the albino mutant, except POR. This increased expression of most genes might be a feedback regulation. POR is a light dependent key enzyme required for chlorophyll biosynthesis by catalyzing protochlorophyllide to chlorophyllide (41, 42). It has been reported that POR is crucial for plant growth and development since nonfunctional plants displayed reduced chlorophyll content and severe photoautotrophic growth defects $(43,44)$. The expression level of POR is lower in albino mutant than in wild type, indicating that albino mutant might be incapable in chlorophyll biosynthesis. MEP pathway is essential for the biosynthesis of photosynthesisrelated compounds, such as carotenoids, chlorophylls, gibberellins and abscisic acid, which is vital important for plant development and metabolism(45). The mutation of genes in the pathway impaired biosynthesis of these compounds, disrupted chloroplast development, resulted in abnormal plant architecture, especially in leaf color(46, 47). IspD (CMS), IspE (CMK) and IspF (MCS) are the third, fourth and fifth enzyme in MEP pathway respectively (Fig. 5b). Related mutants possessed yellow or albino leaves and arrested development of chloroplasts(48-50). In this study, expression of CMS, CMK and MCS in albino mutant was lower than in wild type. The low-level expression may contribute to disruption of chloroplast development. Thylakoid related genes corresponded to reaction centers of PS I and PS II where photochemical reactions occur and convert light energy into chemical energy 31 . Almost all genes related with thylakoid were down-regulated in albino mutant, demonstrating the decrease of photosynthesis viability.

Isocitrate lyase and malate synthase are two unique enzymes to glyoxylate cycle, which was considered essential for postgerminative growth and seedling establishment (51). Under normal condition, glyoxylate cycle enzyme activities decreased rapidly as seedlings become photosynthetic but maintained a continuously high level when grown in dark (52-54). In this study, the isocitrate lyase (Csa2G420990) and malate synthase (Csa1G050360) are abundant in mutant than in wild type (Fig. 6). Wild type started autotrophic growth at this timepoint already, with a high activity of peroxidase (Csa4G285740) and chlorophyll A-B binding protein (Csa3G664560), while albino mutant still needed to utilize the storage because it is still not able to photosynthesize resulted from a lack of functional chloroplast. Glutathione S-transferase have been reported mainly function in response to biotic and abiotic stresses, such as oxidative stress $(55)$, high/low temperature stress $(56,57)$ and different pathogen invasion $(58-60)$. As well, a high concentration of glutathione S-transferase resulted in decreased chlorophyll content (61). When albino mutant is exposed to light, a lethal factor rather than a gift, the mutant presented (Csa4G304240, Csa4G303170) (Fig. 6) more glutathione S-transferase than wild type, this might alsopromote chlorophyll degradation.

Since all the seeds were planted under the same culture condition, we could exclude the possibility of environmental but genetic determination of this albino phenotype. The genetic analysis revealed that this 
albino phenotype was recessively controlled by a single locus. To trace the mutation origin, the previous generation of g32 was also evaluated. Phenotypic evaluation presented no albino performance in all the tested seedlings (data not shown), indicating that the mutation most probably occurred in previous generation in heterozygosis and albinism emerged in this generation. Albinism is not a desired phenomenon in plant breeding since it could affect plant growth as well as production. However, this mutant is of great importance for us to detect new gene that involve in protoplast development.

\section{Conclusions}

In this study, a novel naturally occurring albino mutant was firstly reported in cucumber. We showed that the albino mutant had abnormal chloroplast and did not produce any chlorophyll, therefore, the whole seedling presented white color. Interestingly, we found that this mutant is different from the ones in other plant species, since this cucumber albino mutant could recover white cotyledon to green under dark condition. In addition, with transcriptome data, we detected that differentially expressed genes involved in chlorophyll metabolism, Methylerythritol 4-phosphate (MEP) pathway, as well as thylakoid development. Taken together, this mutant can be a new material to study protoplast development.

\section{Methods}

\section{Plant materials}

Cucumber inbred line g32 was used in this study. The inbred line was provied by Vegetable Research Institute, Guangdong Academy of Agricultural Sciences. The institute is also in responsible for the plant materials used in this study. Yu'e Lin undertakes the formal identification of the plant material. This material has not been deposited in any publicly herbarium. Seeds from a self-pollinated g32 cucumber fruit were soaked into water for 4 hours and then kept in the incubator with moderate humidity at $28^{\circ} \mathrm{C}$ for germination. Thereafter, germinated seeds were planted in plug tray in the greenhouse of Vegetable Research Institute, Guangdong Academy of Agricultural Sciences, Guangzhou, China. Dark treatment was performed in a black plastic bag covered homemade growth chamber. Seven-day-old wild type and albino mutant seedlings were used for phenotypic evaluation, fluorescence microscopy, transmission electron microscopy analysis and high-throughput RNA sequencing.

\section{Fluorescence microscopy}

The abaxial epidermises of cotyledons were used for fluorescence observation. The autofluorescence (red) of chloroplasts were captured under Zeiss LSM710 confocal microscope at the following setting: excitation at $633 \mathrm{~nm}$, emission at 647-721 nm. Data were analyzed using ZEN2010 software.

Transmission electron microscope(TEM)

Cotyledons of wild type and albino mutant seedlings grown under light condition, and cotyledons of albino mutant seedling grown in dark were analyzed for TEM. All the cotyledon samples were cut into 1- 
$2 \mathrm{~mm}^{2}$ sections and fixed in $2.5 \%$ glutaraldehyde and $4 \%$ paraformaldehyde in phosphate buffer $(\mathrm{pH} 6.8-$ 7.2) under a vacuum for 3 hours, followed by washing with phosphate buffer. Subsequently, samples were fixed in $1 \%$ osmium tetroxide for 3 hours, followed by washing with phosphate buffer, and dehydrated in a graduated ethanol series. The samples were infiltrated with an increasing ratio of Spurr's resin dilutions $(25 \%, 50 \%, 75 \%$, and $100 \%(\mathrm{v} / \mathrm{v}))$ to substitute ethanol. Finally, samples were embedded on Spurr's resin. After cutting, the sections were viewed under a HitachiH-7700 (Hitachi) transmission electron microscope.

RNA extraction

Total RNA was extracted from cotyledons of 7-day-old wild type and albino mutant seedlings using Trizol Kit (Promega, USA) according to the manufacturer's instructions. Extracted RNA was treated with RNasefree DNase I (TaKaRa, Japan) to remove residual DNA. RNA degradation and contamination was monitored on $1 \%$ agarose gels. RNA purity was checked using the NanoPhotometer ${ }^{\circledR}$ spectrophotometer (IMPLEN, CA, USA) and RNA integrity was assessed using the RNA Nano 6000 Assay Kit of the Bioanalyzer 2100 system (Agilent Technologies, CA, USA).

cDNA library construction, high-throughput sequencing and mapping

A total amount of $1 \mu \mathrm{g}$ RNA per sample was used as input material for the RNA sample preparations. Sequencing libraries were generated using NEBNext® UltraTM RNA Library Prep Kit for Illumina ${ }^{\circledR}$ (NEB, USA) following manufacturer's recommendations. Library preparations were sequenced on an Illumina Novaseq platform and 150 bp paired-end reads were generated. Thereafter, reads with adaptors, reads with unknown bases as well as low quality reads were removed to generate clean reads. The remaining high-quality clean reads were mapped to Cucumber (Chinese Long) Reference Genome v2 (http://www.cucurbitgenomics.org/organism/2).

Quantification of gene expression, gene ontology (GO), and KEGG pathway enrichment analysis

The mapped reads of each sample were assembled by StringTie (v1.3.3b) 17 in a reference-based approach, and featureCounts v1.5.0-p3 18 was used to count the reads numbers mapped to each gene. FPKM (Fragments Per Kilobase of transcript sequence per Millions base pairs) of each gene was calculated based on the length of the gene and reads count mapped to this gene. Differential expression analysis of two groups was performed using the DESeq2 R package (1.16.1) 19. The resulting P-values were adjusted using the Benjamini and Hochberg's approach for controlling the false discovery rate. Genes with an adjusted P-value (padj) $<0.05$ and $\mid \log 2$ (FoldChange) $\mid>2$ were assigned as differentially expressed genes (DEGs). To functionally annotate detected DEGs, GO (Gene Ontology, http://www.geneontology.org/) and KEGG (Kyoto Encyclopedia of Genes and Genomes, http://www.genome.jp/kegg/) annotation of the unigenes were analyzed using clusterProfiler R package.

Quantitative real-time PCR validation 
To confirm RNA-seq results, 18 DEGs with |log2(FoldChange)| > 3 were randomly selected for qRT-PCR validation. First strand cDNA synthesis was performed using TransScript All-in-One First-Strand cDNA Synthesis SuperMix for qPCR (Transgen, China) with $1 \mu \mathrm{g}$ of RNA as that used for high-throughput sequencing. Quantitative RT-PCR was carried out using 0.2 $\mathrm{\mu g}$ cDNA using PerfectStart Green qPCR Supermix (Transgen, China) according to the instructions. Reaction was performed and analyzed on a CFX96 Real-Time PCR Detection System. Three biological replicates and three technical replicates per sample were performed for each gene. Gene expression was normalized against a-TUBULIN (TUA) gene(62). Primers used were listed in Additional file 1: Table S1.

\section{Abbreviations}

CDS: Coding domain sequence

DEG: Differentially expressed gene

GO: Gene ontology

MEP: Methylerythritol 4-phosphate

PSI: Photosystem I

PSII; Photosystem II

qPCR: Quantitative real-time PCR

TEM: Transmission electron microscope

\section{Declarations}

\section{Ethics approval and consent to participate}

Not applicable.

\section{Consent for publication}

Not applicable.

\section{Availability of data and materials}

The datasets supporting the conclusions of this article are included either within the manuscript or its additional files.

\section{Competing interests}

The authors declare that they have no conflict of interest. 


\section{Funding}

This study was supported by the Presidential foundation of Guangdong Academy of Agricultural Sciences (BZ201901), the Key Laboratory Open Fund Project of Vegetable Institution of GAAS (1000020), Special Fund for Scientific Innovation Strategy-construction of High Level Academy of Agriculture Sciences (R2017PY-JX003, R2018QD-033), Special Fund for Agro-scientific Research in the Public Interest (201503110-07). The funding bodies have no role in the study design, data analysis and interpretation, and manuscript writing, but just provide the financial supports.

\section{Authors' contributions}

J.Y., B.L., X.H., Y.L. and B.J. conceived the research plans; J.Y., Z.C. and P.S. performed most of the experiments; Z.L., W.L., M.W., Q.P. and L.C performed rest of experiments; J.Y. and B.L. analyzed the data; J.Y. and B.L. drafted the paper, X.H., Y.L. and B.J. revised the paper. All authors have read and approved the manuscript.

\section{Acknowledgements}

Not Applicable.

\section{Authors' information}

Jinqiang Yan and Bin Liu contributed equally to this work.

Affiliations

Vegetable Research Institute, Guangdong Academy of Agricultural Sciences, Guangzhou, 510640, China Jinqiang Yan, Zhenqiang Cao, Piaoyun Sun, Wenrui Liu, Zhaojun Liang, Min Wang, Qingwu Peng, Lin Chen, Yu'e Lin, Biao Jiang and Xiaoming He

Guangdong Key Laboratory for New Technology Research of Vegetables, Guangzhou, 510640, China Jinqiang Yan, Piaoyun Sun, Wenrui Liu, Min Wang, Lin Chen, Biao Jiang and Xiaoming He

School of Agriculture and Biology, Shanghai Jiao Tong University, Key Laboratory of Urban Agriculture (South), Ministry of Agriculture, Dongchuan Road, Shanghai, 200240, China

Bin Liu

Corresponding author

Correspondence to Yu'e Lin, Biao Jiang and Xiaoming He.

\section{References}


1. Pogson BJ, Ganguly D, Albrecht-Borth V. Insights into chloroplast biogenesis and development. Biochimica et biophysica acta. 2015;1847(9):1017-24.

2. Chan KX, Phua SY, Crisp P, McQuinn R, Pogson BJ. Learning the Languages of the Chloroplast: Retrograde Signaling and Beyond. Annual review of plant biology. 2016;67:25-53.

3. Unlu C, Drop B, Croce R, van Amerongen H. State transitions in Chlamydomonas reinhardtii strongly modulate the functional size of photosystem II but not of photosystem I. Proceedings of the National Academy of Sciences of the United States of America. 2014;111(9):3460-5.

4. Wang C, Xu W, Jin H, Zhang T, Lai J, Zhou X, et al. A Putative Chloroplast-Localized $\mathrm{Ca}(2+) / \mathrm{H}(+)$ Antiporter CCHA1 Is Involved in Calcium and pH Homeostasis and Required for PSII Function in Arabidopsis. Molecular plant. 2016;9(8):1183-96.

5. Shi K, Gu J, Guo H, Zhao L, Xie Y, Xiong H, et al. Transcriptome and proteomic analyses reveal multiple differences associated with chloroplast development in the spaceflight-induced wheat albino mutant mta. PloS one. 2017;12(5):e0177992.

6. Xiong D, Huang J, Peng S, Li Y. A few enlarged chloroplasts are less efficient in photosynthesis than a large population of small chloroplasts in Arabidopsis thaliana. Scientific reports. 2017;7(1):5782.

7. Gotoh E, Suetsugu N, Yamori W, Ishishita K, Kiyabu R, Fukuda M, et al. Chloroplast Accumulation Response Enhances Leaf Photosynthesis and Plant Biomass Production. Plant physiology. 2018;178(3):1358-69.

8. Zeng $\mathrm{X}$, Tang $\mathrm{R}$, Guo $\mathrm{H}, \mathrm{Ke} \mathrm{S}$, Teng $\mathrm{B}$, Hung $\mathrm{YH}$, et al. A naturally occurring conditional albino mutant in rice caused by defects in the plastid-localized OsABCl8 transporter. Plant molecular biology. 2017;94(1-2):137-48.

9. Wang Y, Zhang J, Shi X, Peng Y, Li P, Lin D, et al. Temperature-sensitive albino gene TCD 5, encoding a monooxygenase, affects chloroplast development at low temperatures. Journal of experimental botany. 2016;67(17):5187-202.

10. Qiu Z, Kang S, He L, Zhao J, Zhang S, Hu J, et al. The newly identified heat-stress sensitive albino 1 gene affects chloroplast development in rice. Plant science : an international journal of experimental plant biology. 2018;267:168-79.

11. Su N, Hu ML, Wu DX, Wu FQ, Fei GL, Lan Y, et al. Disruption of a rice pentatricopeptide repeat protein causes a seedling-specific albino phenotype and its utilization to enhance seed purity in hybrid rice production. Plant physiology. 2012;159(1):227-38.

12. Ruppel NJ, Logsdon CA, Whippo CW, Inoue K, Hangarter RP. A mutation in Arabidopsis seedling plastid development1 affects plastid differentiation in embryo-derived tissues during seedling growth. Plant physiology. 2011;155(1):342-53.

13. Lu M, Han J, Zhu B, Jia H, Yang T, Wang R, et al. Significantly increased amino acid accumulation in a novel albino branch of the tea plant (Camellia sinensis). Planta. 2019;249(2):363-76.

14. Ma Q, Li H, Zou Z, Arkorful E, Lv Q, Zhou Q, et al. Transcriptomic analyses identify albino-associated genes of a novel albino tea germplasm 'Huabai 1'. Horticulture research. 2018;5:54. 
15. Garcia-Alcazar M, Gimenez E, Pineda B, Capel C, Garcia-Sogo B, Sanchez S, et al. Albino T-DNA tomato mutant reveals a key function of 1-deoxy-D-xylulose-5-phosphate synthase (DXS1) in plant development and survival. Scientific reports. 2017;7:45333.

16. Zhao DS, Zhang CQ, Li QF, Yang QQ, Gu MH, Liu QQ. A residue substitution in the plastid ribosomal protein L12/AL1 produces defective plastid ribosome and causes early seedling lethality in rice. Plant molecular biology. 2016;91(1-2):161-77.

17. lida S, Amano E. Mutants induced by pollen irradiation in cucumber. Cucurbit Genet. Coop. Rpt. 1991;14:32-3.

18. Huang S, Li R, Zhang Z, Li L, Gu X, Fan W, et al. The genome of the cucumber, Cucumis sativus $L$. Nature genetics. 2009;41(12):1275-81.

19. Li Z, Zhang Z, Yan P, Huang S, Fei Z, Lin K. RNA-Seq improves annotation of protein-coding genes in the cucumber genome. BMC genomics. 2011;12:540.

20. Cordoba E, Salmi M, Leon P. Unravelling the regulatory mechanisms that modulate the MEP pathway in higher plants. Journal of experimental botany. 2009;60(10):2933-43.

21. Qin D, Dong J, Xu F, Guo G, Ge S, Xu Q, et al. Characterization and fine mapping of a novel barley Stage Green-Revertible Albino Gene (HvSGRA) by Bulked Segregant Analysis based on SSR assay and Specific Length Amplified Fragment Sequencing. BMC genomics. 2015;16:838.

22. Lv Y, Shao G, Qiu J, Jiao G, Sheng Z, Xie L, et al. White Leaf and Panicle 2, encoding a PEPassociated protein, is required for chloroplast biogenesis under heat stress in rice. Journal of experimental botany. 2017;68(18):5147-60.

23. He L, Zhang S, Qiu Z, Zhao J, Nie W, Lin H, et al. FRUCTOKINASE-LIKE PROTEIN 1 interacts with TRXz to regulate chloroplast development in rice. Journal of integrative plant biology. 2018;60(2):94-111.

24. Yin T, Pan G, Liu H, Wu J, Li Y, Zhao Z, et al. The chloroplast ribosomal protein $L 21$ gene is essential for plastid development and embryogenesis in Arabidopsis. Planta. 2012;235(5):907-21.

25. Lin D, Jiang Q, Zheng K, Chen S, Zhou H, Gong X, et al. Mutation of the rice ASL2 gene encoding plastid ribosomal protein L21 causes chloroplast developmental defects and seedling death. Plant biology (Stuttgart, Germany). 2015;17(3):599-607.

26. Huang $X$, Zhang $X$, Yang S. A novel chloroplast-localized protein EMB1303 is required for chloroplast development in Arabidopsis. Cell research. 2009;19(10):1205-16.

27. Liang Q, Lu X, Jiang L, Wang C, Fan Y, Zhang C. EMB1211 is required for normal embryo development and influences chloroplast biogenesis in Arabidopsis. Physiologia plantarum. 2010;140(4):380-94.

28. Ye LS, Zhang Q, Pan H, Huang C, Yang ZN, Yu QB. EMB2738, which encodes a putative plastidtargeted GTP-binding protein, is essential for embryogenesis and chloroplast development in higher plants. Physiologia plantarum. 2017;161(3):414-30.

29. Chen H, Li S, Li L, Hu H, Zhao J. Arabidopsis EMB1990 Encoding a Plastid-Targeted YlmG Protein Is Required for Chloroplast Biogenesis and Embryo Development. Frontiers in plant science. 2018;9:181. 
30. Qin G, Gu H, Ma L, Peng Y, Deng XW, Chen Z, et al. Disruption of phytoene desaturase gene results in albino and dwarf phenotypes in Arabidopsis by impairing chlorophyll, carotenoid, and gibberellin biosynthesis. Cell research. 2007;17(5):471-82.

31. Kakizaki T, Matsumura H, Nakayama K, Che FS, Terauchi R, Inaba T. Coordination of plastid protein import and nuclear gene expression by plastid-to-nucleus retrograde signaling. Plant physiology. 2009;151(3):1339-53.

32. Shanmugabalaji V, Chahtane H, Accossato S, Rahire M, Gouzerh G, Lopez-Molina L, et al. Chloroplast Biogenesis Controlled by DELLA-TOC159 Interaction in Early Plant Development. Current biology : CB. 2018;28(16):2616-23.e5.

33. Zhang T, Feng P, Li Y, Yu P, Yu G, Sang X, et al. VIRESCENT-ALBINO LEAF 1 regulates leaf colour development and cell division in rice. Journal of experimental botany. 2018;69(20):4791-804.

34. Kamaraj B, Purohit R. Mutational analysis of oculocutaneous albinism: a compact review. BioMed research international. 2014;2014:905472.

35. Galante Rocha de Vasconcelos FT, Hauzman E, Dutra Henriques L, Kilpp Goulart PR, de Faria Galvao $\mathrm{O}$, Sano RY, et al. A novel nonsense mutation in the tyrosinase gene is related to the albinism in a capuchin monkey (Sapajus apella). BMC genetics. 2017;18(1):39.

36. Le Ret M, Belcher S, Graindorge S, Wallet C, Koechler S, Erhardt M, et al. Efficient Replication of the Plastid Genome Requires an Organellar Thymidine Kinase. Plant physiology. 2018;178(4):1643-56.

37. Zhu X, Liang S, Yin J, Yuan C, Wang J, Li W, et al. The DnaJ OsDjA7/8 is essential for chloroplast development in rice (Oryza sativa). Gene. 2015;574(1):11-9.

38. Wang Y, Wang C, Zheng M, Lyu J, Xu Y, Li X, et al. WHITE PANICLE1, a Val-tRNA Synthetase Regulating Chloroplast Ribosome Biogenesis in Rice, Is Essential for Early Chloroplast Development. Plant physiology. 2016;170(4):2110-23.

39. Schneider J, Labory CR, Rangel WM, Alves E, Guilherme LR. Anatomy and ultrastructure alterations of Leucaena leucocephala (Lam.) inoculated with mycorrhizal fungi in response to arseniccontaminated soil. Journal of hazardous materials. 2013;262:1245-58.

40. Steiner S, Schroter Y, Pfalz J, Pfannschmidt T. Identification of essential subunits in the plastidencoded RNA polymerase complex reveals building blocks for proper plastid development. Plant physiology. 2011;157(3):1043-55.

41. Grubler B, Merendino L, Twardziok So, Mininno M, Allorent G, Chevalier F, et al. Light and Plastid Signals Regulate Different Sets of Genes in the Albino Mutant Pap7-1. Plant physiology. 2017;175(3):1203-19.

42. Yang J, Cheng Q. Origin and evolution of the light-dependent protochlorophyllide oxidoreductase (LPOR) genes. Plant biology (Stuttgart, Germany). 2004;6(5):537-44.

43. Zhang S, Heyes DJ, Feng L, Sun W, Johannissen LO, Liu H, et al. Structural basis for enzymatic photocatalysis in chlorophyll biosynthesis. Nature. 2019;574(7780):722-5.

44. Kim C, Apel K. Arabidopsis light-dependent NADPH: protochlorophyllide oxidoreductase A (PORA) is essential for normal plant growth and development: an addendum. Plant molecular biology. 
2012;80(2):237-40.

45. Paddock T, Lima D, Mason ME, Apel K, Armstrong GA. Arabidopsis light-dependent protochlorophyllide oxidoreductase A (PORA) is essential for normal plant growth and development. Plant molecular biology. 2012;78(4-5):447-60.

46. Flores-Perez U, Sauret-Gueto S, Gas E, Jarvis P, Rodriguez-Concepcion M. A mutant impaired in the production of plastome-encoded proteins uncovers a mechanism for the homeostasis of isoprenoid biosynthetic enzymes in Arabidopsis plastids. The Plant cell. 2008;20(5):1303-15.

47. Xing S, Miao J, Li S, Qin G, Tang S, Li H, et al. Disruption of the 1-deoxy-D-xylulose-5-phosphate reductoisomerase (DXR) gene results in albino, dwarf and defects in trichome initiation and stomata closure in Arabidopsis. Cell research. 2010;20(6):688-700.

48. Chandran AKN, Lee GS, Yoo YH, Yoon UH, Ahn BO, Yun DW, et al. Functional classification of rice flanking sequence tagged genes using MapMan terms and global understanding on metabolic and regulatory pathways affected by dxr mutant having defects in light response. Rice (New York, NY). 2016;9(1):17.

49. Hsieh MH, Chang CY, Hsu SJ, Chen JJ. Chloroplast localization of methylerythritol 4-phosphate pathway enzymes and regulation of mitochondrial genes in ispD and ispE albino mutants in Arabidopsis. Plant molecular biology. 2008;66(6):663-73.

50. Chen N, Wang P, Li C, Wang Q, Pan J, Xiao F, et al. A Single Nucleotide Mutation of the IspE Gene Participating in the MEP Pathway for Isoprenoid Biosynthesis Causes a Green-Revertible Yellow Leaf Phenotype in Rice. Plant \& cell physiology. 2018;59(9):1905-17.

51. Huang R, Wang Y, Wang P, Li C, Xiao F, Chen N, et al. A single nucleotide mutation of IspF gene involved in the MEP pathway for isoprenoid biosynthesis causes yellow-green leaf phenotype in rice. Plant molecular biology. 2018;96(1-2):5-16.

52. Eastmond PJ, Germain V, Lange PR, Bryce JH, Smith SM, Graham IA. Postgerminative growth and lipid catabolism in oilseeds lacking the glyoxylate cycle. Proceedings of the National Academy of Sciences of the United States of America. 2000;97(10):5669-74.

53. Trelease RN, Becker WM, Gruber PJ, Newcomb EH. Microbodies (Glyoxysomes and Peroxisomes) in Cucumber Cotyledons: Correlative Biochemical and Ultrastructural Study in Light- and Dark-grown Seedlings. Plant physiology. 1971;48(4):461-75.

54. Becker WM, Leaver CJ, Weir EM, Riezman H. Regulation of Glyoxysomal Enzymes during Germination of Cucumber: I. Developmental Changes in Cotyledonary Protein, RNA, and Enzyme Activities during Germination. Plant physiology. 1978;62(4):542-9.

55. He D, Damaris RN, Fu J, Tu J, Fu T, Xi C, et al. Differential Molecular Responses of Rapeseed Cotyledons to Light and Dark Reveal Metabolic Adaptations toward Autotrophy Establishment. Frontiers in plant science. 2016;7:988.

56. Horvath E, Bela K, Papdi C, Galle A, Szabados L, Tari I, et al. The role of Arabidopsis glutathione transferase F9 gene under oxidative stress in seedlings. Acta biologica Hungarica. 2015;66(4):40618. 
57. Roxas VP, Smith RK, Jr., Allen ER, Allen RD. Overexpression of glutathione S-transferase/glutathione peroxidase enhances the growth of transgenic tobacco seedlings during stress. Nature biotechnology. 1997;15(10):988-91.

58. Liang D, Gao F, Ni Z, Lin L, Deng Q, Tang Y, et al. Melatonin Improves Heat Tolerance in Kiwifruit Seedlings through Promoting Antioxidant Enzymatic Activity and Glutathione S-Transferase Transcription. Molecules (Basel, Switzerland). 2018;23(3).

59. Sytykiewicz H, Chrzanowski G, Czerniewicz P, Sprawka I, Lukasik I, Golawska S, et al. Expression profiling of selected glutathione transferase genes in Zea mays (L.) seedlings infested with cereal aphids. PloS one. 2014;9(11):e111863.

60. Gong Q, Yang Z, Chen E, Sun G, He S, Butt HI, et al. A Phi-Class Glutathione S-Transferase Gene for Verticillium Wilt Resistance in Gossypium arboreum Identified in a Genome-Wide Association Study. Plant \& cell physiology. 2018;59(2):275-89.

61. Ducker R, Zollner P, Lummen P, Ries S, Collavo A, Beffa R. Glutathione transferase plays a major role in flufenacet resistance of ryegrass (Lolium spp.) field populations. Pest management science. 2019;75(11):3084-92.

62. Jiang HW, Liu MJ, Chen IC, Huang CH, Chao LY, Hsieh HL. A glutathione S-transferase regulated by light and hormones participates in the modulation of Arabidopsis seedling development. Plant physiology. 2010;154(4):1646-58.

63. Liu B, Liu X, Yang S, Chen C, Xue S, Cai Y, et al. Silencing of the gibberellin receptor homolog, CsGID1a, affects locule formation in cucumber (Cucumis sativus) fruit. The New phytologist. 2016;210(2):551-63.

\section{Additional File Information}

Additional file 1: Table S1. Primers used for qRT-PCR validation.

Additional file 2: Table S2. Segregation ratio of albino and wild type seedlings in progenies of g32.

Additional file 3: Table S3. Up-regulated and down-regualted genes in albino mutant.

Additional file 4: Table S4. GO enrichment analysis of differentially expressed genes between albino mutant and wild type.

Additional file 5: Table S5. KEGG analysis of differentially expressed genes between albino mutant and wild type.

Additional file 6: Table S6. Differentially expressed genes in chlorophyll metabolism and MEP pathway between albino mutant and wild type.

Additional file 7: Table S7. Differentially expressed genes of reported albino genes between albino mutant and wild type. 
Additional file 8: Figure S1. Histogram of GO enrichment analysis.

\section{Table}

Table 1. RNA sequencing reads and sequencing quality of all samples

\begin{tabular}{llllllll}
\hline Sample & Raw_reads & Clean_reads & Clean_bases & Error_rate & Q20 & Q30 & GC_pct \\
\hline Albino-1 & $112,074,500$ & $110,556,516$ & $8.29 \mathrm{G}$ & 0.03 & 97.37 & 92.56 & 43.31 \\
Albino_2 & $115,086,664$ & $113,466,224$ & $8.51 \mathrm{G}$ & 0.03 & 97.47 & 92.73 & 43.43 \\
\hline Albino_3 & $116,871,728$ & $115,589,940$ & $8.67 \mathrm{G}$ & 0.03 & 97.47 & 92.77 & 43.45 \\
\hline Normal_1 & $102,760,028$ & $101,241,304$ & $7.59 \mathrm{G}$ & 0.03 & 97.5 & 92.83 & 43.16 \\
\hline Normal_2 & $127,644,424$ & $123,516,412$ & $9.62 \mathrm{G}$ & 0.03 & 97.43 & 92.68 & 43.43 \\
\hline Normal_3 & $100,197,308$ & $97,869,948$ & $7.34 \mathrm{G}$ & 0.03 & 97.35 & 92.54 & 43.55 \\
\hline
\end{tabular}

\section{Figures}

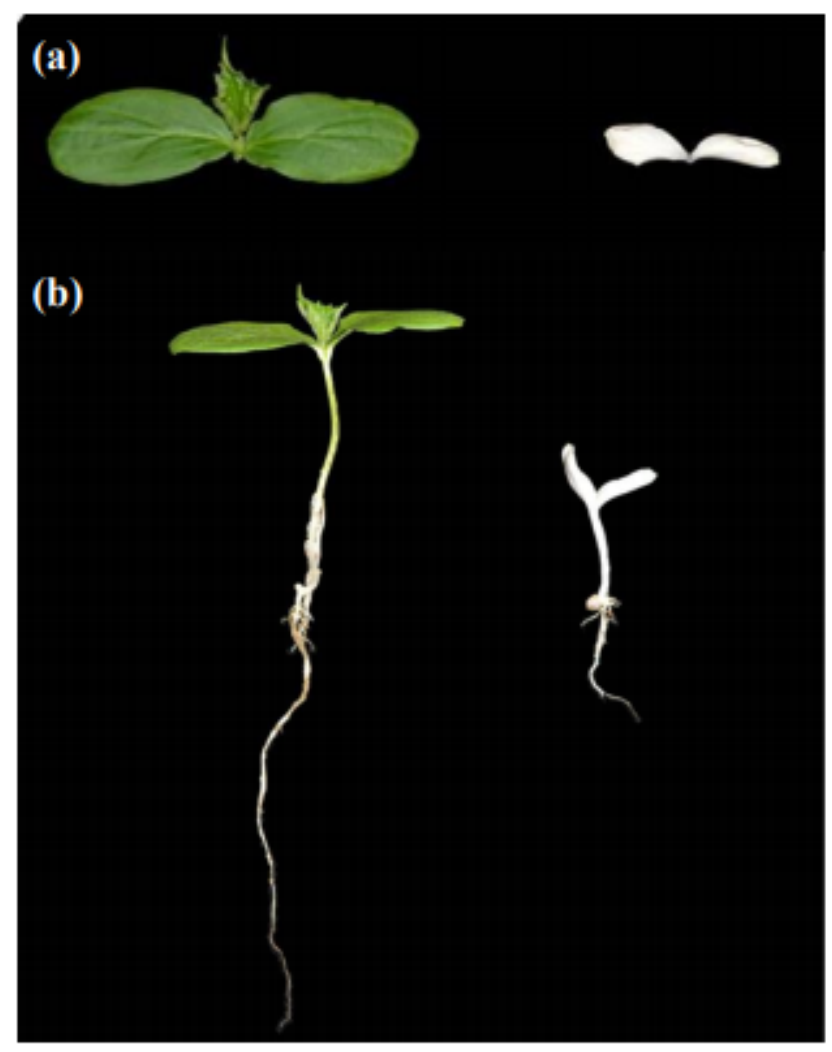

Figure 1 
Growth phenotype of albino mutant and wild type from the progenies of a cucumber inbred line g32. a Differences in cotyledons of albino mutant and wild type seedlings. b Morphological difference between albino mutant and wild type seedlings.
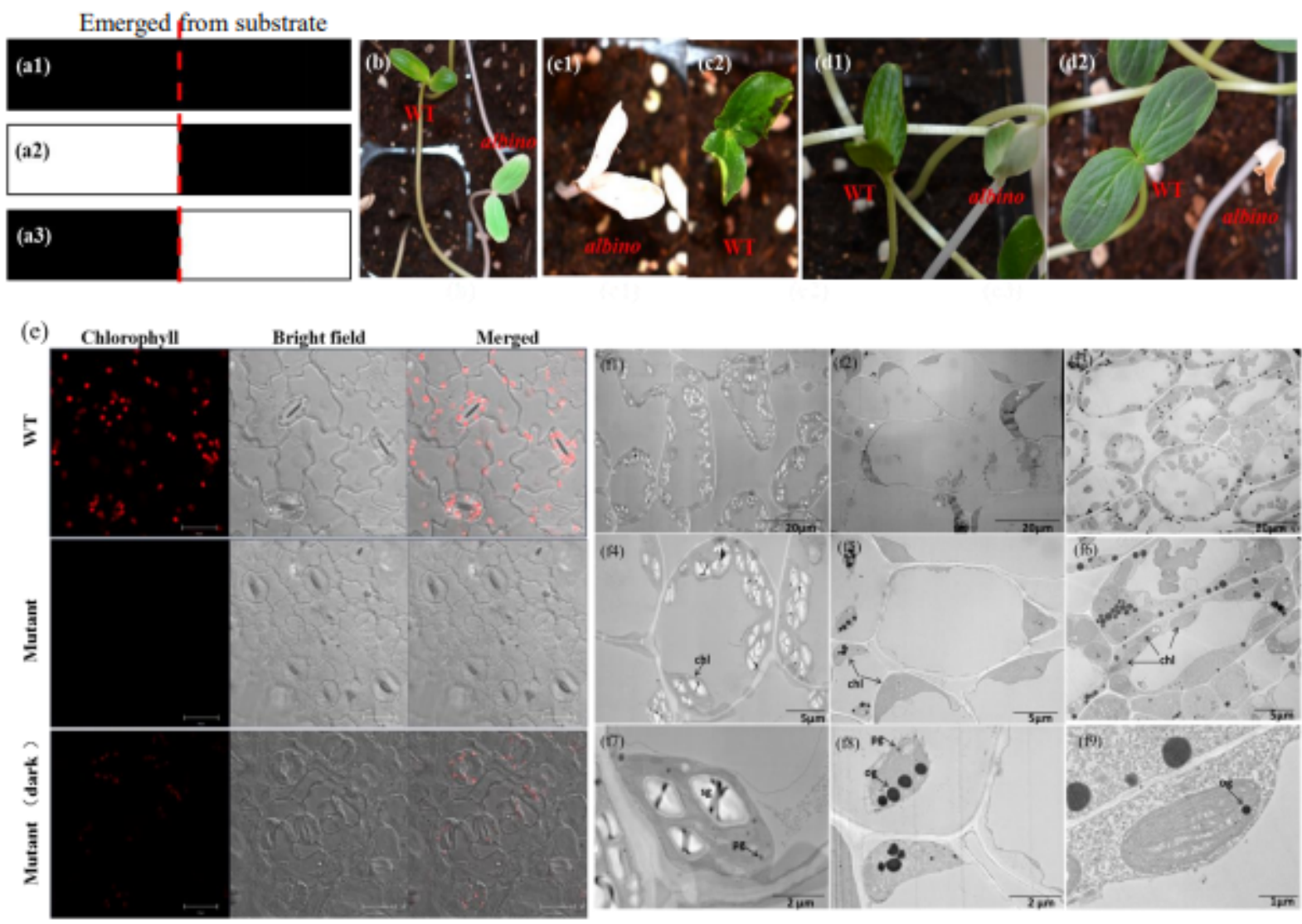

\section{Figure 2}

Short-lived chloroplast recovery in albino mutant under dark condition. a1-a3 Schematic illustration of light and dark treatment of WT and albino seedlings. a1 Plants were treated with continuous dark before and after they emerged from substrate. a2 First, plants were treated with light, then after emerging from substrate, they were moved to dark. a3 First, plants were treated with dark, then after emerging from substrate, they were moved to light condition. b Phenotype of WT and albino seedlings under indicated condition that described in a1. c Phenotype of WT (c1) and albino (c2) seedlings in the indicated conditions that described in a2. d Phenotype of WT and albino seedlings in the indicated conditions that described in a3. d1 Phenotype of WT and albino seedlings exposed to light after 5 hours. d2 Phenotype of WT and albino seedlings exposed to light after 30 hours. e Fluorescence microscopy images of cotyledon abaxial epidermis of wild type, albino mutant cucumber seedlings grown under light condition and albino

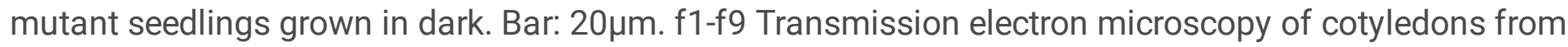
albino mutant and wild type seedlings. $\mathrm{f} 1, \mathrm{f} 4$ An overview of cotyledon cells of wild type grown under light condition. $f 2$, f5 An overview of cotyledon cells of albino mutant grown under light condition. $f 3, f 6$ An overview of cotyledon cells of albino mutant grown in dark. f7, f8, f9 Enlarged views of chloroplast ultrastructure of wild type, albino mutant growon under light condition and albino mutant grown in dark, respectively. chl, chloroplast; sg, starch granules; pg, plastoglobuli; og, osmiophilic plastoglobuli. 

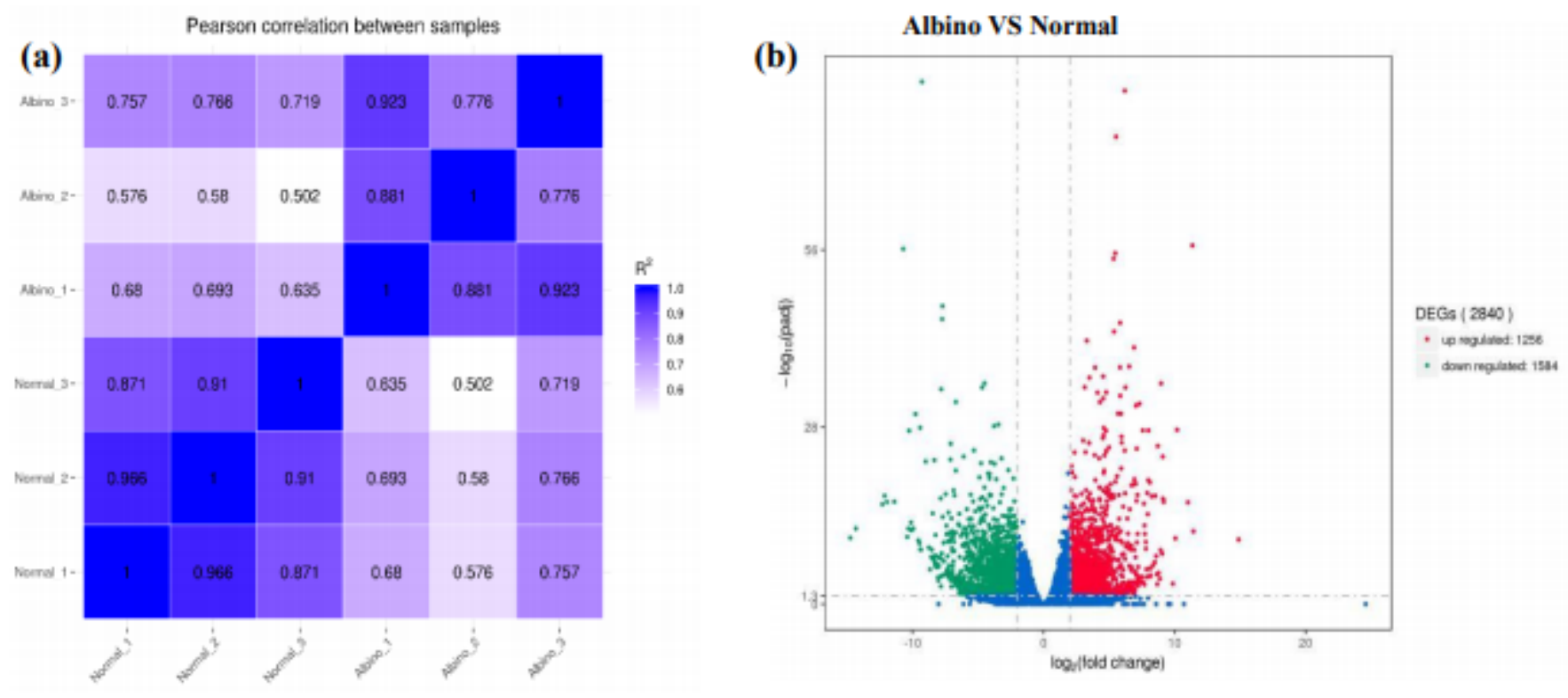

Figure 3

Diagrams illustrating correlations/distances among transcriptomes and the number of differentially expressed genes in albino mutant and wild type seedlings. a Correlation matrix and cluster dendrogram of the whole dataset of the mapped reads. The analysis was performed by comparing the values of the entire transcriptome in all two samples with three biological replicates. Correlation analysis (coefficients R2) and hierarchical cluster analysis were performed using R software. Dark blue color indicates a stronger correlation and light blue weaker (R2). b Volcano plot showing DEGs between albino mutant and wild type. X-axis represents log2 Fold Change and y-axis represents -log10 (padj). Red, green and blue dots represents up-regulated, down-regulated and not DEGs, respectively. 


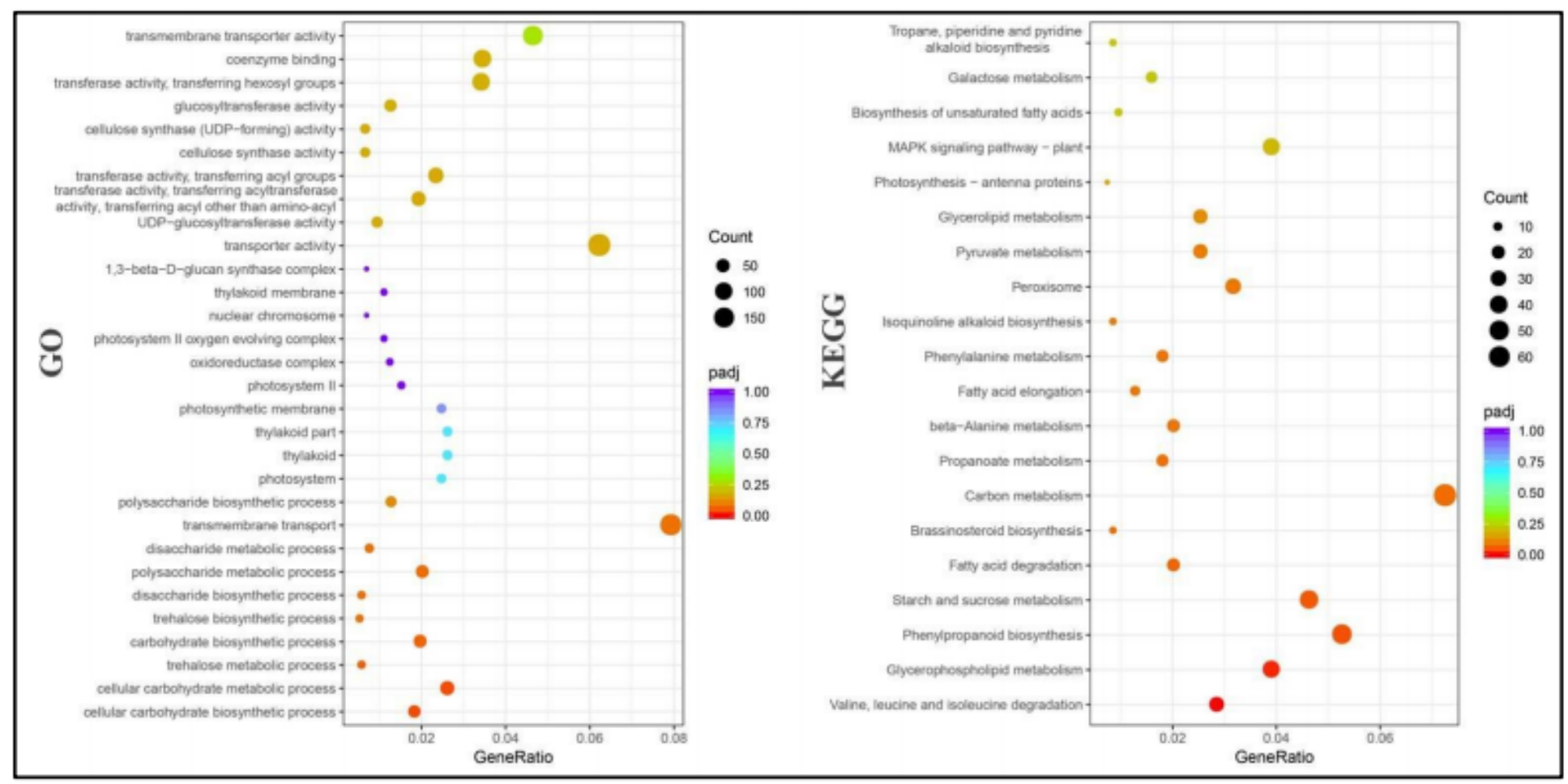

Figure 4

Genes enriched in different categories in the GO (left) and KEGG (right) analysis. X-axis represents the gene ratio of enriched genes among the background genes in different categories and while y-axis represents the GO or KEGG terms. A high padj-value is represented by red, and a low value is represented by purple. The size of the bubble represents the number of genes annotated to the terms. 
(a)

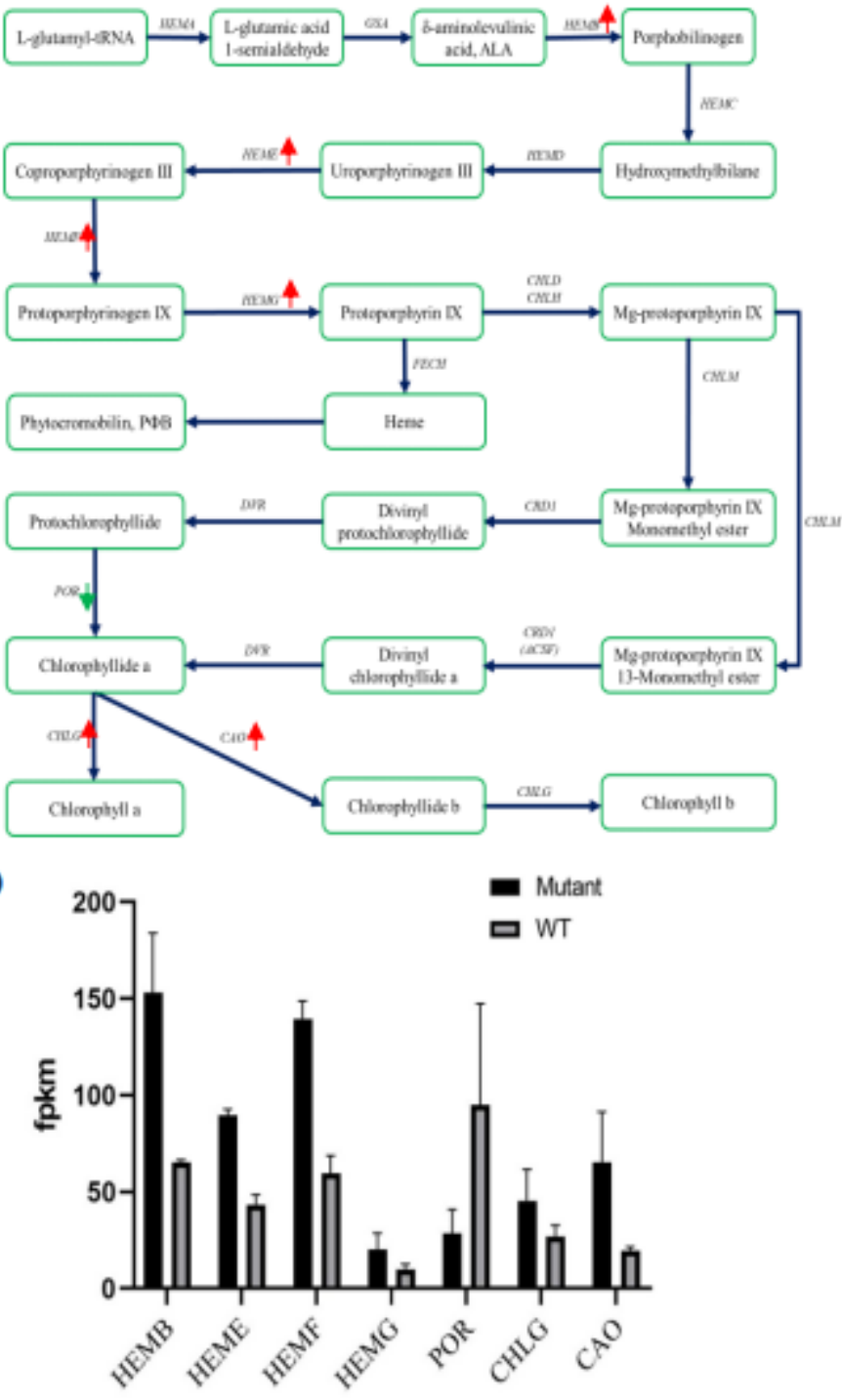

(b)GAP

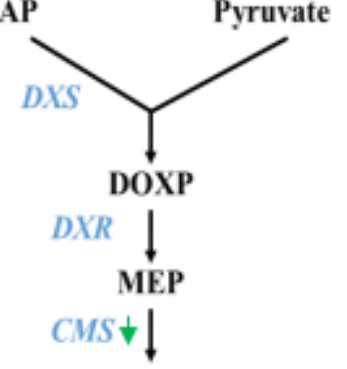

CDP-ME

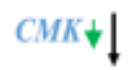

CDP-MEP

$M C S \downarrow \downarrow$

ME-cPP

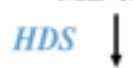

HMBPP

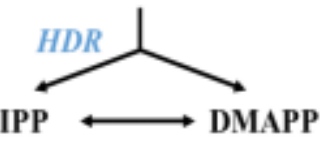

(d)

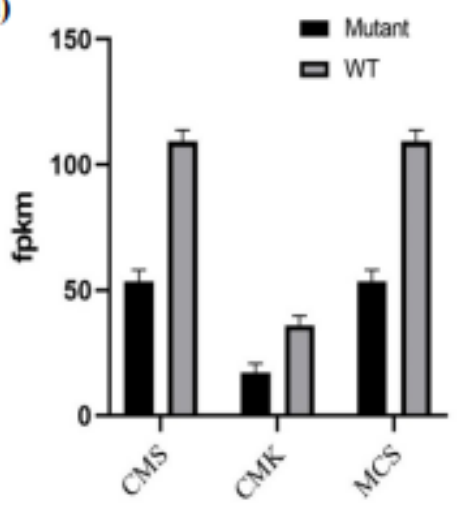

Figure 5

Differentially expression of genes associated with chlorophyll metabolism and Methylerythritol 4phosphate (MEP) pathway. a, b Diagram showing major genes in chlorophyll metabolism and MEP pathway, respectively. Red arrows indicate up-regulated genes while green arrows indicate down-regulated genes in albino mutant. c, d Expression profile of DEGs in chlorophyll metabolism and MEP pathway achieved by RNA-seq. The mean FPKM (fragments per kilobase of transcript per million mapped reads) values for the DEGs were calculated from three biological replicates for each genotype. The error bars indicate the standard deviations. GAP, glyceraldehyde-3-phosphate; DOXP, 1-deoxy-D-xylulose-5phosphate; MEP, 2-C-methyl-D-erythritol-4-phosphate; CDP-ME, 4-diphosphocytidyl-2-C-methyl-D-erythritol; CDP-MEP, 4-diphosphocytidyl-2-C-methyl-D-erythritol-2-phosphate; ME-cPP, 2-C-methyl-D-erythritol-2,4-Ccyclodiphosphate; HMBPP, 1-hydroxy-2-methyl-2-(E)-butenyl-4-diphosphate; IPP, isopentenyl diphosphate; DMAPP, dimethylallyl diphosphate. 

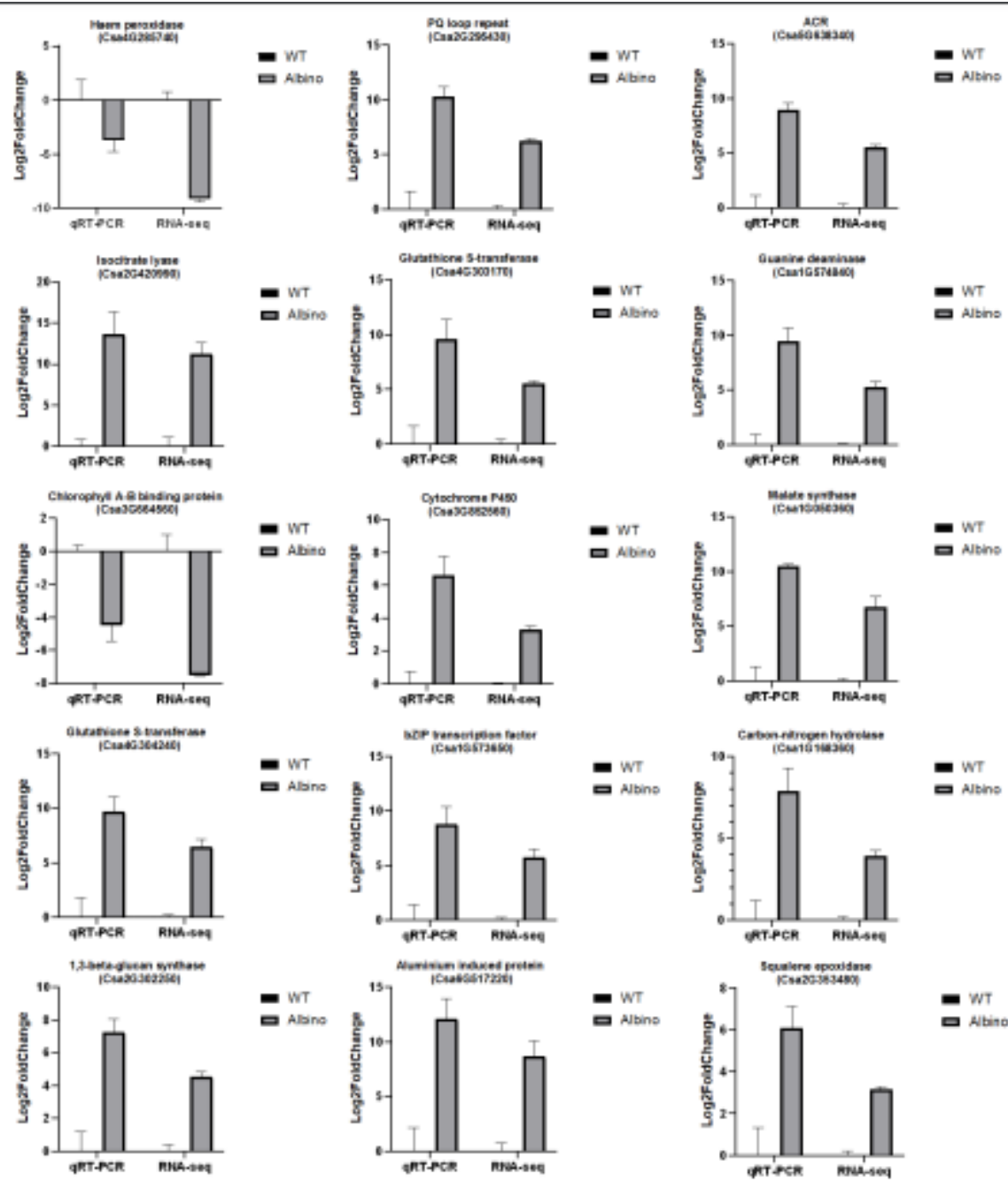

a
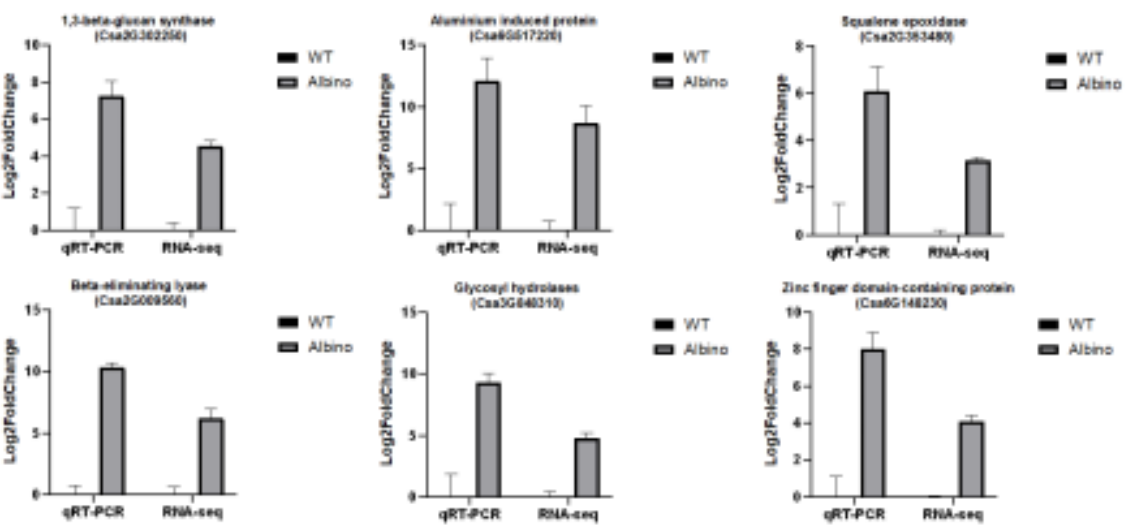

Figure 6

Expression profile of selected differentially expressed genes between albino mutant and wild type from RNA-seq result achieved by qRT-PCR. X-axis represents gene name while y-axis represents relative expression $(-\Delta \Delta C t)$ value of each gene. Data are shown as means $(n=3)$. The error bars indicate the standard deviations

\section{Supplementary Files}

This is a list of supplementary files associated with this preprint. Click to download.

- Additionalfile5.xls

- Additionalfile1.doc 
- Additionalfile8.doc

- Additionalfile7.xls

- Additionalfile6.xls

- Additionalfile3.xls

- Additionalfile2.doc

- Additionalfile4.xls 